Eur. J. Clin. Chem. Clin. Biochem.

Vol. 30, 1992, pp. 209-216

(C) 1992 Walter de Gruyter \& Co.

Berlin $\cdot$ New York

\title{
Simultaneous Detection of Whole Blood Chemiluminescence in Microtitre Plates
}

\author{
By V. Kaever ${ }^{1}, J . T$. Robitzsch ${ }^{1}, W$. Stangel ${ }^{2}$, L. Schleinkofer $^{3}$ and $K$. Resch ${ }^{1}$ \\ ${ }^{1}$ Institut für Molekularpharmakologie, Medizinische Hochschule Hannover, Hannover, Germany \\ ${ }^{2}$ Institut für Transfusionsmedizin und Immunhämatologie, Medizinische Hochschule Hannover, Hannover, \\ Germany \\ ${ }^{3}$ Hamamatsu Photonics, Herrsching, Germany
}

(Received June 5, 1991/January 15, 1992)

Summary: The measurement of reactive oxygen species provides a simple method for monitoring the degree of activation of leukocytes in various disorders, and for determining the effects of drugs on this activation. The present report describes the determination of luminol- or lucigenin-amplified chemiluminescence of whole blood in a microtitre plate assay with a 96-well luminometer (HAMAMATSU MTP reader $^{\circledR}$ ).

Using heparinized venous human blood from healthy donors, optimal chemiluminescence intensities were determined at a blood dilution of $1 / 100$ in a total volume of $0.25 \mathrm{ml}$ of Hank's balanced salt solution, containing $0.4 \mathrm{mmol} / 1$ luminol as enhancer and either opsonized zymosan $(1 \mathrm{~g} / \mathrm{l})$ or the phorbol ester, $12-\mathrm{O}$ tetradecanoylphorbol 13 -acetate $\left(10^{-6} \mathrm{~mol} / \mathrm{l}\right)$, as stimuli. The in vitro effects of nordihydroguaiaretic acid, diphenylene iodonium, and diclofenac were tested. After preincubation of the diluted whole blood with these drugs for $15 \mathrm{~min}$, the zymosan-stimulated chemiluminescence was diminished in all cases. The specific NADPH oxidase inhibitor, diphenylene iodonium, was most effective (half maximal inhibition at $1.5 \times 10^{-8} \mathrm{~mol} / \mathrm{l}$ ), whereas higher concentrations of the antioxidant, nordihydroguaiaretic acid $\left(1.6 \times 10^{-6} \mathrm{~mol} / \mathrm{l}\right)$, or the nonsteroidal antiinflammatory drug, diclofenac (about $10^{-5} \mathrm{~mol} / \mathrm{l}$ ), were needed to achieve half maximal inhibition.

In addition to its usefulness in the rapid screening of drug effects this assay system seems to be very beneficial for the clinical diagnosis of congenital disorders. Furthermore, it is suited as an effective and simple method for the continuous determination of the phagocyte functional state in patients in pathophysiological situations and during therapy.

\section{Introduction}

Reactive oxygen species such as the superoxide anion radical $\left(\mathrm{O}_{2}^{-}\right)$, hydroxyl radicals, hydrogen peroxide $\left(\mathrm{H}_{2} \mathrm{O}_{2}\right)$, and hypochlorous acid (generated by the myeloperoxidase-mediated conversion of $\mathrm{H}_{2} \mathrm{O}_{2}$ in the pres-

\footnotetext{
1) Enzymes:

Catalase $\left(\mathrm{H}_{2} \mathrm{O}_{2}: \mathrm{H}_{2} \mathrm{O}_{2}\right.$ oxidoreductase, EC 1.11.1.6)

Myeloperoxidase (myeloperoxidase, EC 1.11.1.7)

NADPH oxidase (NADPH : $\mathrm{O}_{2}$-oxidoreductase)

Superoxide dismutase (superoxide: superoxide oxidoreductase, EC 1.15.1.1)

Xanthine oxidase (xanthine: oxygen oxidoreductase, EC 1.1.3.22)
}

ence of halide) contribute to the development of cytotoxic and antimicrobial activities of activated phagocytes (1) and also play an important role in inflammatory processes (2). Various methods for the measurement of reaction oxygen species have been established (3). For example, $\mathrm{O}_{2}^{-}$can be determined by the superoxide dismutase ${ }^{1}$ )-inhibitable reduction of cytochrome $\mathrm{c}$ or nitroblue tetrazolium salts (4) and $\mathrm{H}_{2} \mathrm{O}_{2}$ by reaction with phenol red (4) or scopoletin (5). Reactive oxygen species decay is accompanied by the emission of small amounts of light but this chemiluminescence is enormously intensified in the presence of chemical amplifiers such as luminol or lucigenin 
(6). As this method is very simple, provided that a suitable measuring instrument is available, the detection of chemiluminescence is used widely as a functional quantity of polymorphonuclear leukocytes and monocytic cells (7).

We (8) and others (9) have previously described an assay system for the detection of enhanced chemiluminescence of isolated phagocytes in microtitre plates. In this report we show that by using an even more sensitive luminometer (10) the chemiluminescence of diluted whole blood can also be evaluated in 96 samples monitored in parallel, using only micro-quantities of human blood.

\section{Materials and Methods}

\section{Materials}

All assays are performed in white 96-well microtitre plates (MicroFLUOR ${ }^{\circledast}$, Dynatech, Denkendorf, Germany) using a luminometer equipped with a sensitive single photon imaging device (MTP reader ${ }^{\circledR}$, Hamamatsu Photonics, Herrsching, Germany). This system allows the continuous chemiluminescence registration of all wells simultaneously. The chemical enhancers, luminol (5'-amino-2,3-dihydro-1,4-phthalazinedione) and lucigenin $\left(\left[9,9^{\prime}\right.\right.$-bis-( $\mathrm{N}$-methyl-acridinium nitrate) $\left.]\right)$, were obtained from Boehringer, Mannheim, Germany, and stored at $4{ }^{\circ} \mathrm{C}$ as $10^{-2} \mathrm{~mol} / 1$ stock solutions in Hank's balanced salt solution (Gibco, Eggenstein, Germany). When luminol was used, triethanolamine ( $5 \mathrm{ml} / 1$ Hank's balanced salt solution) was also added to achieve solution. The stimuli, 12-O-tetradecanoylphorbol 13acetate $\left(10^{-3} \mathrm{~mol} / 1\right.$ stock solution in dimethylsulphoxide at $-80^{\circ} \mathrm{C}$ ) and zymosan, were purchased from Sigma (Deisenhofen, Germany). Nordihydroguaiaretic acid $\left(10^{-2} \mathrm{~mol} / 1\right.$ stock solution in ethanol at $\left.-20^{\circ} \mathrm{C}\right)$, diclofenac $\left(10^{-3} \mathrm{~mol} / \mathrm{l}\right.$ stock solution in Hank's balanced salt solution in $\left.-20^{\circ} \mathrm{C}\right)$, sodium azide, superoxide dismutase (from bovine erythrocytes, $3 \mathrm{kU} /$ $\mathrm{mg}$ protein), and catalase (from bovine liver, $44 \mathrm{kU} / \mathrm{mg}$ protein) were also from Sigma. Diphenylene iodonium was kindly provided by Prof. O. T. G. Jones (Bristol, U. K.).

\section{Methods}

Venous blood was taken from healthy adults and coagulation was prevented by addition of $10 \cdot 10^{3} \mathrm{U} / 1$ heparin (Liquemin ${ }^{\circledR}$ N 25000, Hoffmann-La Roche, Grenzach-Wyhlen, Germany). Leukocyte counts and the haemoglobin content were determined in the clinical routine laboratory by a Coulter ${ }^{\circledR}$ Counter system.

After storage of the whole blood at room temperature for no longer than two hours in polypropylene tubes, it was diluted in Hank's balanced salt solution directly before the start of the experiment.

Opsonized zymosan was prepared as follows. Zymosan $(1 \mathrm{ml}$, $50 \mathrm{~g} / \mathrm{l}$ ) was boiled in phosphate-buffered saline for $90 \mathrm{~min}$ and washed three times with phosphate buffered saline by centrifugation $(16000 \mathrm{~g}, 10 \mathrm{~min})$. The final pellet was resuspended in $20 \mathrm{ml}$ pooled human serum and incubated for $30 \mathrm{~min}$ at $37^{\circ} \mathrm{C}$. After washing with phosphate buffered saline, the opsonized zymosan $(10 \mathrm{~g} / \mathrm{l})$ was stored at $-20^{\circ} \mathrm{C}$. Chemiluminescence measurements were performed in a total volume of $250 \mu \mathrm{l} /$ well. Whole blood $(50 \mu \mathrm{l}$, diluted $1 / 20$ with Hank's balanced salt solution) was carefully mixed with enhancer $(100 \mu \mathrm{l})$ and Hank's balanced salt solution $(75 \mu \mathrm{l})$ and preincubated for $30 \mathrm{~min}$ at $37^{\circ} \mathrm{C}$ in the luminometer. After addition of the stimulus or vehicle $(25 \mu \mathrm{l})$ light emission was continuously recorded for 30 $\mathrm{min}$. For the investigation of drug effects on reactive oxygen species formation, nordihydroguaiaretic acid, diphenylene iodonium, diclofenac, superoxide dismutase, catalase, or sodium azide were included in the assay $15 \mathrm{~min}$ prior to the stimulus addition.

\section{Results}

Optimization of assay conditions for whole blood chemiluminescence measurement in 96-well microtitre plates

Experiments were first carried out to determine the optimal assay conditions for whole blood chemiluminescence measurement in the 96-well luminometer. The sensitivity of the assay largely depends on the choice of a suitable chemical chemiluminescence enhancer. Figure 1 shows the effects of luminol and lucigenin, respectively, on opsonized zymosan- or 12O-tetradecanoylphorbol 13-acetate-stimulated emission over $30 \mathrm{~min}$ from 1/100 diluted whole blood. With both stimuli significantly higher total counts were observed when luminol was included, showing an optimum at a concentration of about $400 \mu \mathrm{mol} / \mathrm{l}$. Lucigenin revealed its best enhancing effect on light emission at lower concentrations (about $100 \mu \mathrm{mol} / \mathrm{l}$ ). The stimulation of reactive oxygen species production was more pronounced with opsonized zymosan than with 12-O-tetradecanoylphorbol 13-acetate for both enhancers. This holds true for total chemiluminescence recordings (fig. 1) as well as for the maximal chemiluminescence intensities seen at about $10 \mathrm{~min}$ after stimulus addition (data not shown). Therefore, further experiments were performed solely in the presence of luminol.

Next we evaluated the dose-dependency of the stimuli. As can be seen in figure 2, the highest stimulatory effects were observed using opsonized zymosan in concentrations between 0.5 and $1.5 \mathrm{~g} / \mathrm{l}$, or $12-\mathrm{O}$-tetradecanoylphorbol 13 -acetate at $10^{-6}$ to $10^{-5} \mathrm{~mol} / \mathrm{l}$. Non-opsonized zymosan was about half as effective as opsonized zymosan in its ability to induce reactive oxygen species production (data not shown). Further experiments were done in the presence of either opsonized zymosan $(1 \mathrm{~g} / \mathrm{l})$ or 12-O-tetradecanoylphorbol 13-acetate $\left(10^{-6} \mathrm{~mol} / \mathrm{l}\right)$.

The most critical point in whole blood chemiluminescence measurement is the dilution of the heparinized whole blood, in order to reduce the concentrations of substances that interfere with reactive oxygen species production or quench light emission. Using blood from healthy donors a distinct maximum of light emission after stimulation with opsonized zymosan or 12-O-tetradecanoylphorbol 13-acetate was 

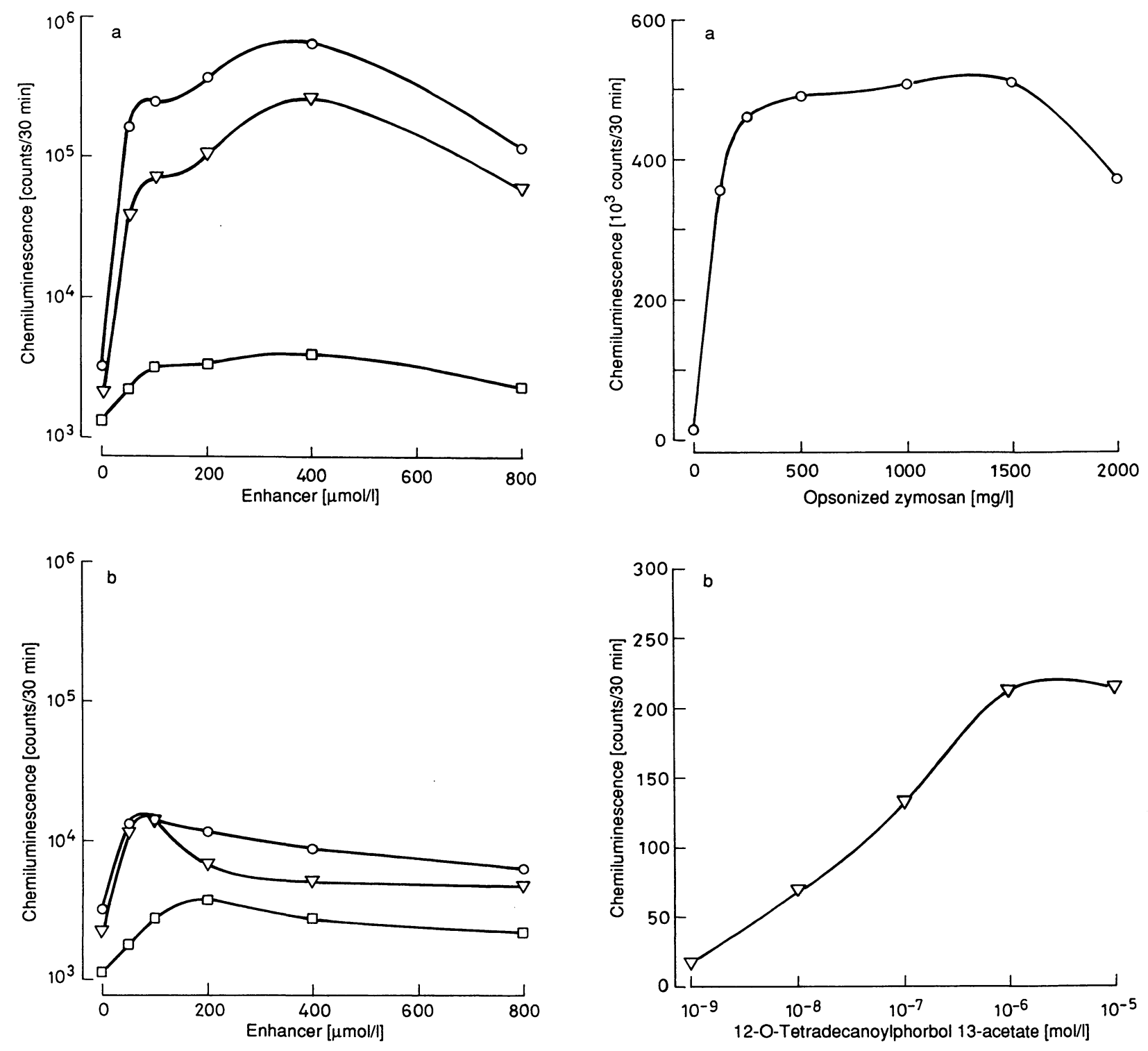

Fig. 1. Determination of the most suitable enhancer concentration for whole blood chemiluminescence. Blood (finally diluted 1/100) was preincubated with luminol (fig. 1a) or lucigenin (fig. 1b) at the indicated concentrations and stimulated by the addition of either opsonized zymosan $(1 \mathrm{~g} / \mathrm{l})(0)$, the phorbol ester 12-O-tetradecanoylphorbol 13-acetate $\left(10^{-6} \mathrm{~mol} / \mathrm{l}\right)(\nabla)$, or Hank's balanced salt solution ( $\square$ ). Total counts integrated over $30 \mathrm{~min}$ are presented. The background value without enhancers was always less than 1000 counts $/ 30 \mathrm{~min}$. Data are the mean of duplicate cell incubations from one representative experiment out of three similar ones.

seen at a final blood dilution of $1 / 100$ (fig. 3). Assuming a mean leukocyte count in blood of $5 \cdot 10^{9} / 1$ only about 12500 leukocytes were present in each well in a total volume of $250 \mu \mathrm{l}$.

In routine assays the diluted blood was preincubated at $37^{\circ} \mathrm{C}$ in the luminometer with luminol for $30 \mathrm{~min}$ prior to the stimulation. The simultaneous addition of enhancer and stimuli resulted in an unchanged total

Fig. 2. Determination of the most suitable stimulus concentration for whole blood chemiluminescence. Diluted blood $(1 / 100)$ was preincubated with luminol $\left(4 \times 10^{-4} \mathrm{~mol} / \mathrm{l}\right)$ and stimulated by the addition of either opsonized zymosan (Fig. 2a, O) or 12-O-tetradecanoylphorbol 13-acetate (Fig. 2b, $\nabla$ ) at the indicated concentrations. Data are presented as described in fig. 1.

light emission (area under the curve) or maximal intensity, but the maximum of reactive oxygen species production was shifted to even earlier time points (data not shown). It is therefore recommended that the preincubation time should be held constant (i.e. $30 \mathrm{~min}$ ) during all experiments.

Influence of different effectors on reactive oxygen species production in whole blood

In order to further characterize our assay system the effects of different substances interfering with the 


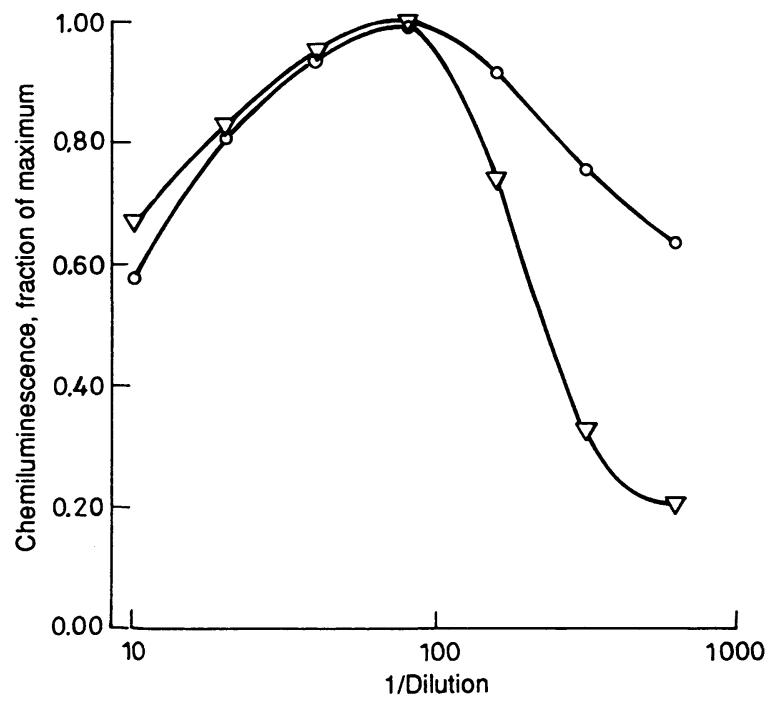

Fig. 3. Determination of the most suitable blood dilution for stimulated chemiluminescence measurement. Whole blood in different dilutions as indicated was preincubated with luminol $\left(4 \times 10^{-4} \mathrm{~mol} / \mathrm{l}\right)$ and stimulated by the addition of either opsonized zymosan $(1 \mathrm{~g} / \mathrm{l})(\mathrm{O})$ or 12-O-tetradecanoylphorbol 13-acetate $\left(10^{-6} \mathrm{~mol} / \mathrm{l}\right)(\nabla)$. Data are presented as described in fig. 1 .

reactive oxygen species production were investigated. In these experiments it proved to be absolutely necessary to treat control cells and stimulated cells in an identical manner. For example, addition of Hank's balanced salt solution buffer alone led to an up to tenfold enhancement of total chemiluminescence generation. In all experiments the effectors were preincubated for $15 \mathrm{~min}$ with the diluted whole blood and the chemical enhancer before stimulus addition and the start of the 30 min chemiluminescence recording period. The specific NADPH oxidase inhibitor, diphenylene iodonium, effectively diminished light emission from whole blood. Half maximal inhibition of basal or opsonized zymosan-induced reactive oxygen species production was noted at a concentration of $1.5 \times 10^{-8} \mathrm{~mol} / \mathrm{l}$ and no chemiluminescence was recorded at a concentration of $1 \times 10^{-6} \mathrm{~mol} / \mathrm{l}$. A similar effect was achieved with sodium azide, which at $10^{-4}$ mol/l nearly totally $(90 \%)$ blocked reactive oxygen species production (data not shown). Addition of superoxide dismutase $(1 \mathrm{MU} / \mathrm{l})$ decreased the measured chemiluminescence less effectively $(75 \%$ inhibition). The effects of catalase were very complex in the whole blood system. At a low catalase concentration $(20 \mathrm{kU} / \mathrm{l})$ the basal and 12-O-tetradecanoylphorbol 13-acetate-stimulated reactive oxygen species production was slightly enhanced, whereas at higher concentrations of catalase $(500-1000 \mathrm{kU} / \mathrm{l})$ a $50 \%$ inhibition of the opsonized zymosan-stimulated chemiluminescence was observed (data not shown).

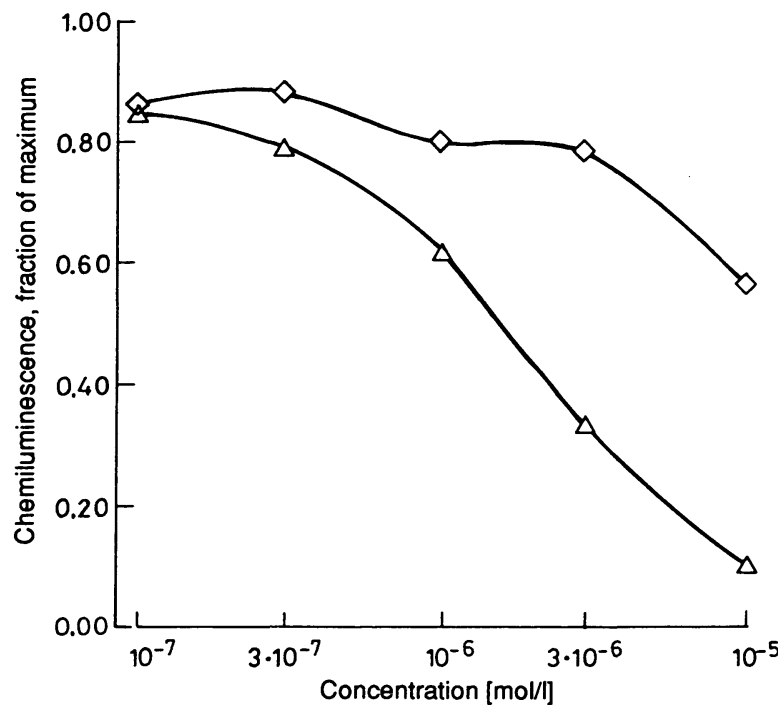

Fig. 4. In vitro drug effects on whole blood chemiluminescence. Diluted blood (1/100) was preincubated with luminol $\left(4 \times 10^{-4} \mathrm{~mol} / \mathrm{l}\right)$ and either diclofenac $(\diamond)$ or nordihydroguaiaretic acid $(\triangle)$ at the indicated concentrations as described in Materials and Methods. Stimulation was achieved by addition of opsonized zymosan $(1 \mathrm{~g} / \mathrm{l})$. Total counts integrated over $30 \mathrm{~min}$ as the mean from duplicate cell incubations are given.

In vitro drug effects on opsonized zymosanstimulated reactive oxygen species production in whole blood

Using a procedure similar to that described for effectors in the previous section, the direct effects of several drugs on cellular reactive oxygen species production can easily be followed with the described chemiluminescence system. In a first attempt the influences of the antioxidant nordihydroguaiaretic acid and the non-steroidal antiinflammatory drug diclofenac were tested. In whole blood the opsonized zymosan-induced light emission was dose-dependently depressed by both agents with nordihydroguaiaretic acid showing a half maximal inhibition at a concentration of $1.6 \times 10^{-6} \mathrm{~mol} / 1$ and diclofenac at about $1 \times 10^{-5}$ $\mathrm{mol} / \mathrm{l}$ (fig. 4)

Establishment of reference values for healthy adults

Under optimal assay conditions blood from 177 healthy donors (124 male and 53 female, age 19-63) was used to establish normal values for basal, opsonized zymosan (1 g/l)-, and 12-O-tetradecanoylphorbol 13 -acetate $\left(10^{-6} \mathrm{~mol} / \mathrm{l}\right)$-stimulated chemiluminescence responses. As can be seen in figure 5, maximal intensities obtained after about 10 min correlated very well to the total counts (area under curve) obtained during the whole $30 \mathrm{~min}$ incubation time. Therefore, 

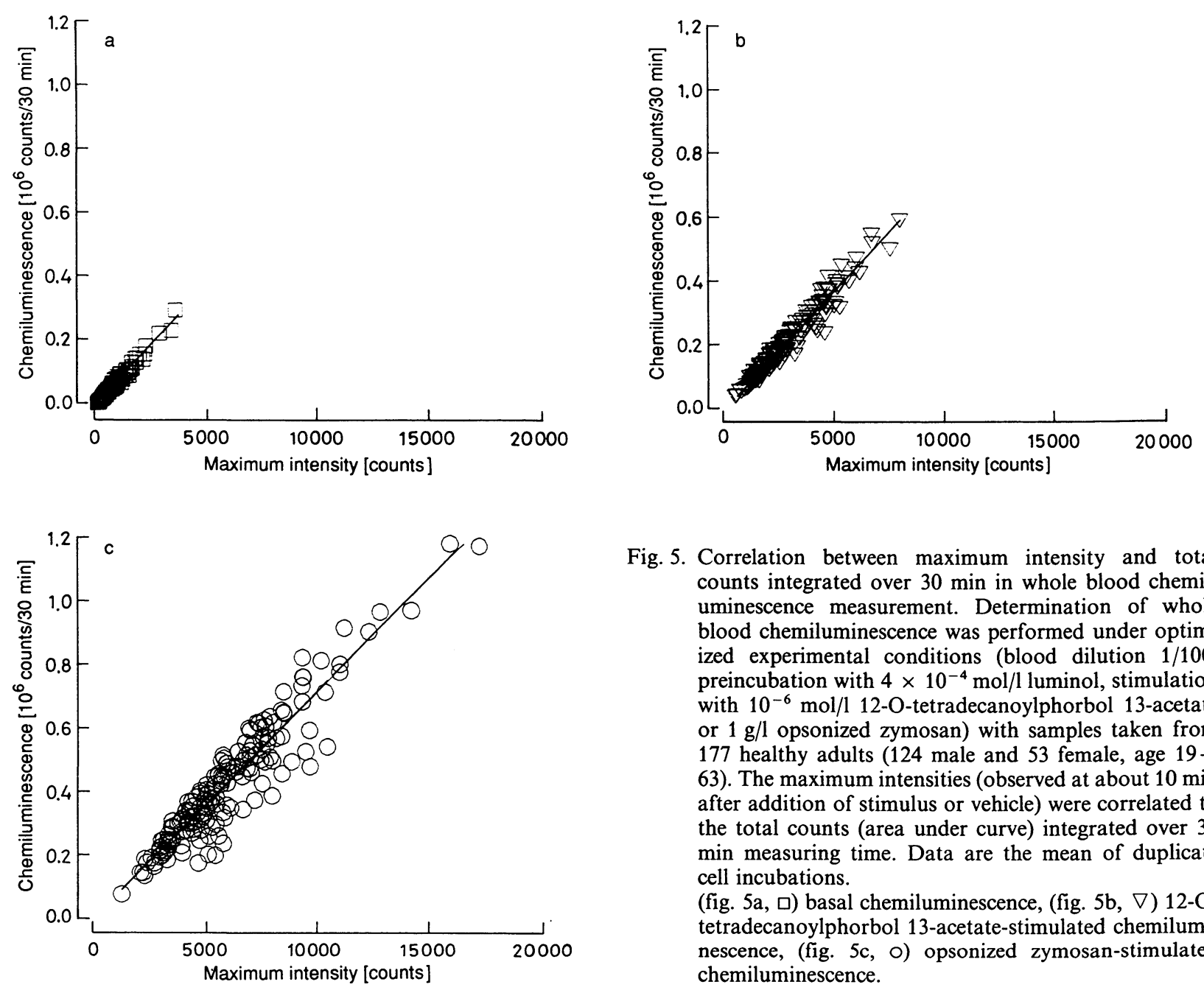

Fig. 5. Correlation between maximum intensity and total counts integrated over $30 \mathrm{~min}$ in whole blood chemiluminescence measurement. Determination of whole blood chemiluminescence was performed under optimized experimental conditions (blood dilution $1 / 100$, preincubation with $4 \times 10^{-4} \mathrm{~mol} / \mathrm{l}$ luminol, stimulation with $10^{-6} \mathrm{~mol} / \mathrm{l} 12$-O-tetradecanoylphorbol 13-acetate or $1 \mathrm{~g} / \mathrm{l}$ opsonized zymosan) with samples taken from 177 healthy adults (124 male and 53 female, age 1963 ). The maximum intensities (observed at about $10 \mathrm{~min}$ after addition of stimulus or vehicle) were correlated to the total counts (area under curve) integrated over 30 min measuring time. Data are the mean of duplicate cell incubations.

(fig. $5 \mathrm{a}, \mathrm{\square}$ ) basal chemiluminescence, (fig. $5 \mathrm{~b}, \nabla)$ 12-Otetradecanoylphorbol 13-acetate-stimulated chemiluminescence, (fig. 5c, O) opsonized zymosan-stimulated chemiluminescence.

Tab. 1. Reference values of whole blood chemiluminescence

\begin{tabular}{lccccc}
\hline & Minimum & Maximum & Mean & S. D. & CV \\
\hline Leukocytes & 2.9 & 14.7 & 5.6 & 1.5 & 26.8 \\
Basal chemiluminescence & & & & \\
$\quad$ Area under curve & 6.7 & 294.0 & 57.4 & 43.0 & 74.9 \\
Area under curve/leukocytes & 0.65 & 22.0 & 4.1 & 3.1 & 76.1 \\
Opsonized zymosan-stimulated chemiluminescence & & & & \\
Area under curve & 38.1 & 588.0 & 205.0 & 111.0 & 54.1 \\
Area under curve/leukocytes & 4.4 & 41.8 & 14.6 & 7.2 & 49.1 \\
12-O-tetradecanoylphorbol 13-acetate-stimulated chemiluminescence & & & \\
Area under curve & 81.8 & 1184.0 & 426.0 & 194.0 & 45.5 \\
Area under curve/leukocytes & 11.3 & 64.8 & 29.8 & 29.8 & 32.9 \\
\hline
\end{tabular}

Whole blood chemiluminescence measurement was performed with samples from 177 healthy adults as specified in figure 5 .

Area under curve, $10^{3}$ counts, Leukocytes, $10^{9} / 1, \mathrm{CV}=$ coefficient of variation.

further data (tab. 1) were calculated on the basis of the total count determinations. Although there was a large range of light emission, especially in the control cells (treated with Hank's balanced salt solution), a pronounced stimulation of reactive oxygen species production was achieved either by the addition of 12 O-tetradecanoylphorbol 13-acetate (mean 3.6 fold) or opsonized zymosan (mean 7.4 fold). When chemiluminescence values were corrected for leukocyte count (tab. 1, area under curve/leukocytes), the deviation of the opsonized zymosan stimulation was clearly diminished from 45.5 to $32.9 \%$. No correlation was found between chemiluminescence values and either the age of the donors or the haemoglobin content of 
the blood samples (data not shown). It has to be noted, however, that only adult blood donors were included in this experimental series and the haemoglobin concentrations were quite similar within the blood samples $(120-160 \mathrm{~g} / \mathrm{l})$. There was also no significant difference in the chemiluminescence values between males and females (data not shown).

\section{Discussion}

Measurement of whole blood chemiluminescence was first described more than ten years ago by Kato et al., who believed that chemiluminescence determination in unfractionated whole blood would become a valuable method for clinical routine diagnosis (11). It seems reasonable that whole blood chemiluminescence provoked by particulate or soluble stimuli is directly correlated with the activation stage of human phagocytes and, therefore, represents an early indicator of the progression of different pathophysiological situations. In addition, it may be used as an effective screening system for drugs that either directly interfere with the phagocyte oxidative burst or exert immunomodulatory effects. The availability of modern luminometers allowing the simultaneous chemiluminescence detection and computerization from several samples has led to a revival of this methodological approach in recent years $(12-15)$. It was the aim of this study to provide evidence that a sensitive 96-well luminometer can be also used for this purpose.

It has always been a matter of debate whether chemiluminescence measurements should be performed with whole blood or isolated blood cells. It is incontestable that reactive oxygen species production strongly depends on the presence and distribution of granulocytes and monocytes in different blood samples and that various blood components, especially erythrocytes (16), interfere with the light emission. In order to minimize such interferences highly diluted blood should be used. In our assay system a final 100 -fold dilution (corresponding to $2.5 \mu \mathrm{l}$ blood per well) resulted in optimum light emission, and higher dilutions with only a few thousand cells per well were also possible. On the other hand, the isolation of mononuclear or polymorphonuclear leukocytes requires time-consuming centrifugation steps, which may lead to preactivation of the cells. The commonly performed lysis of remaining erythrocytes is also difficult to standardize. From these considerations it seems preferable to use whole blood in clinical routine assays and isolated phagocytes in more detailed studies.

Based on our previous observation that whole blood chemiluminescence measurement is practicable in 96- well microtitre plates (8) we first determined the optimum assay conditions and the effects of several agents known to influence the production of reactive oxygen species. It is well documented that activation of the phagocyte NADPH oxidase by various stimuli is associated with increased oxygen consumption, anaerobic glycolysis via the hexose monophosphate shunt, and production of reactive oxygen species (17). In the presence of myeloperoxidase, hydrogen peroxide formed from the superoxide anion radical can be converted to strongly bactericidal halides. In normal blood, the numbers of granulocytes greatly exceed those of other reactive oxygen species-producing cells. It is therefore assumed that whole blood chemiluminescence predominantly depends on the neutrophil myeloperoxidase- $\mathrm{H}_{2} \mathrm{O}_{2}$-halide system (12). This correlates well with our observation that chemiluminescence amplification by lucigenin, which more specifically reacts with $\mathrm{O}_{2}^{-}(18)$, results in a significantly lower light emission than enhancement by luminol, which may be a better bystander substrate for other reactive oxygen species besides $\mathrm{O}_{2}^{-}$. Addition of diphenylene iodonium, a specific inhibitor of the NADPH oxidase (19), totally blocked reactive oxygen species production at very low concentrations. Although sodium azide is known to have no influence on lucigenin-enhanced chemiluminescence from isolated neutrophils (18), in our hands it inhibited the luminol-amplified chemiluminescence from opsonized zymosan-stimulated whole blood. This again emphasizes the importance of the myeloperoxidase system, which is inactivated by azide $(12,20)$. The addition of superoxide dismutase or catalase resulted in either a less effective chemiluminescence decrease, or in more complex effects reflecting the presence of several enzyme systems involved in the reactive oxygen species production in whole blood. Taken together, the results obtained with the 96-well microtitre plate system used in the present study are comparable to those obtained with conventional 1 - or 6-channel luminometers $(12-15)$.

The described assay system seems well-suited for the screening of drug effects on cellular reactive oxygen species production. This holds true for changes induced in vivo or in vitro by biological response modifiers (8), or even more so for the determination of direct drug effects on blood cells, as demonstrated in our study by experiments with the antioxidant, nordihydroguaiaretic acid, or the non-steroidal antiinflammatory drug, diclofenac. Compared with other luminometer systems the outstanding advantage depends on the fact that the effects of several drugs in different concentrations can be simultaneously determined in the same blood sample. In addition, only 
$250 \mu \mathrm{l}$ blood are needed for an assay of 96 samples in one microtitre plate. Meanwhile, we have studied the effects of a broad series of phosphodiesterase inhibitors on whole blood chemiluminescence generation (data will be reported separately). The same advantages are also evident when isolated cells are used for such studies, although whole blood chemiluminescence most probably better agrees with in vivo conditions. It should be noted that in detailed experiments the drug effects should also be tested in a cellfree system (i. e. $\mathrm{O}_{2}^{-}$production by xanthine/xanthine oxidase) in order to estimate unspecific scavenging of reactive oxygen species.

In addition to drug screening, whole blood chemiluminescence measurements have been performed in numerous clinical studies dealing with the evaluation of congenital phagocyte disorders such as chronic granulomatous disease or various pathophysiological situations. Furthermore, an enhanced release of reactive oxygen species from leukocytes may be one important pathogenic factor in asthma, psoriasis, and other inflammatory reactions, acute respiratory distress syndrome, multiple organ failure, and myocardial infarction. It can be assumed that pathophysiological situations involving especially granulocytes can be followed up by monitoring whole blood chemiluminescence. In diseases in which mainly mononuclear leukocytes play a predominant role no changes in whole blood chemiluminescence may be expected.

Especially in the febrile period during a bacterial infection reactive oxygen species production is markedly increased and, therefore, whole blood chemiluminescence measurement may provide an early clinical indicator in sepsis. However, we believe that it is not advisable to determine blood samples from many different patients with various infections, as the necessary controls before the onset of the infection are often lacking. It seems more promising to monitor the progress of the infection and the effect of therapeutic intervention in well-defined clinical situations. Our first study addressing the establishment of normal values for whole blood chemiluminescence from healthy adults has clearly shown, in accordance with other investigations $(12,14,15)$, a high degree of interindividual variation, although blood was only stored for short periods before the assay (13), and correction factors for leukocyte counts and the haemoglobin content were used (13). In our hands no significant difference between samples from males and females was obvious. In preliminary experiments we found that capillary blood could also be used for whole blood chemiluminescence measurements, and the results were similar to those obtained with venous blood. For this purpose a microtitre plate luminometer offers the advantage that samples from several patients can be measured in a short time. In addition, the effects of different stimuli (opsonized zymosan, 12-O-tetradecanoylphorbol 13-acetate, but also complement factors, chemotactic peptides, and cytokines) in various concentrations can be simultaneously determined, which provides additional data for the assessment and further management of the respective pathophysiological situation. We have now started a new clinical study, in which chemiluminescence is measured in arterial and venous whole blood from patients after heart transplantation.

\section{Acknowledgement}

The skilfull technical assistance of Heike Hartmann is gratefully acknowledged.

\section{References}

1. McPhail, L. \& Snyderman, R. (1984) Mechanisms of regulating the respiratory burst of leukocytes. In: Regulation of leukocyte function (Snyderman, R., ed.) pp. 247-281, Plenum Press, New York.

2. Ward, P. A., Johnson, K. J. \& Till, G. O. (1986) Tissue injury caused by toxic oxygen products from phagocytic cells. In: Chemical mediators of inflammation and immunity (Cohen, S., Hayashi, H., Saito, K. \& Takada, A., eds.) pp. 73-83, Academic Press, Tokyo.

3. Weber, G. F. (1990) The measurement of oxygen-derived free radicals and related substances in medicine. J. Clin. Chem. Clin. Biochem. 28, 569-603.

4. Pick, E. (1986) Microassays for superoxide and hydrogen peroxide production and nitroblue tetrazolium reduction using an enzyme immunoassay microplate reader. In: Methods in Enzymology, vol. 132 (Di Sabato, G. \& Everse, J. eds.) pp. 407-421, Academic Press, New York.
5. De la Harpe, J. \& Nathan, C. F. (1985) A semi-automated micro-assay for $\mathrm{H}_{2} \mathrm{O}_{2}$ release by human blood monocytes and mouse peritoneal macrophages. J. Immunol. Methods 78, 323-336.

6. Müller-Peddinghaus, R. (1984) In vitro determination of phagocyte activity by luminol- and lucigenin-amplified chemiluminescence. Int. J. Immunopharmac. 6, 455-466.

7. van Dyke, K. (1987) Introduction to cellular chemiluminescence, neutrophils, macrophages, and monocytes. In: Cellular chemiluminescence (van Dyke, K. \& Castranova, V., eds.) pp. $3-22$, CRC Press, Boca Raton.

8. Kaever, V., Schmitz, E. \& Resch, K. (1990) Evaluation of macrophage activation stage by measurement of lucigeninenhanced chemiluminescence using a 96-well microtiter system. Fresenius J. Anal. Chem. 337, 92-93.

9. Blair, A. L., Cree, I. A., Beck, J. S. \& Hastings, M. J. G. (1988) Measurement of phagocyte chemiluminescence in a microtitre plate format. J. Immunol. Methods 112, $163-$ 168. 
10. Maly, F. E., Urweler, A., Rolli, H. P., Dahinden, C. A. \& de Weck, A. L. (1988) A single-photon imaging system for the simultaneous quantitation of luminescent emissions from multiple samples. Anal. Biochem. 168, 462-469.

11. Kato, T., Wokalek, H., Schöpf, E., Eggert, H., Ernst, M., Rietschel, E. Th. \& Fischer, H. (1981) Measurement of chemiluminescence in freshly drawn human blood. 1. Role of granulocytes, platelets, and plasma factors in zymosaninduced chemiluminescence. Klin. Wochenschr. 59, 203211.

12. Lindena, J., Burkhardt, H. \& Dwenger, A. (1987) Mechanisms of non-opsonized zymosan-induced and luminol-enhanced chemiluminescence in whole blood and isolated phagocytes. J. Clin. Chem. Clin. Biochem. 25, 765- 778.

13. Ristola, M. \& Repo, H. (1989) Luminol-enhanced chemiluminescence of whole blood. APMIS 97, 503-512.

14. Krause, S., Michel, E., Brachmann, P., Lösche, W., Heptinstall, S. \& Till, U. (1989) Untersuchungen der zellulären Chemilumineszenz aktivierter Phagozyten im Vollblut. Folia Haematol., Leipzig 116, 955-965.

15. Parente, R. \& Melotti, C. (1989) Automation and computerization of chemiluminescence: A new methodological approach in the study of human phagocytes. J. Biolumin. Chemilumin. 4, 602-608.
16. Redl, H., Lamche, H. \& Schlag, G. (1983) Red cell count dependence of whole blood granulocyte luminescence. Klin. Wochenschr. 61, 163-164.

17. Hurst, N. P. (1987) Molecular basis of activation and regulation of the phagocyte respiratory burst. Ann. Rheum. Dis. 46, 265-272.

18. Gyllenhammar, H. (1987) Lucigenin chemiluminescence in the assessment of neutrophil superoxide production. J. Immunol. Meth. 97. 209-213.

19. Cross, A. R. \& Jones, O. T. G. (1986) The effect of the inhibitor diphenylene iodonium on the superoxide-generating system of neutrophils. Specific labelling of a component polypeptide of the oxidase. Biochem. J. 237, $111-$ 116.

20. Suematsu, M., Oshio, C. \& Miura, S. (1988) Luminoldependent photoemission from single neutrophil stimulated by phorbol ester and calcium ionophore - Role of degranulation and myeloperoxidase. Biochem. Biophys. Res. Comm. 155, 106-111.

\section{Priv.-Doz. Dr. Volkhard Kaever}

Medizinische Hochschule Hannover Institut für Molekularpharmakologie Konstanty-Gutschow-Straße 8

W-3000 Hannover 61

Bundesrepublik Deutschland 